\title{
Widespread white matter degeneration preceding the onset of dementia
}

\section{Citation}

Maier-Hein, Klaus H., Carl-Fredrik Westin, Martha E. Shenton, Michael W. Weiner, Ashish Raj, Philipp Thomann, Ron Kikinis, Bram Stieltjes, and Ofer Pasternak. 2015. “Widespread White Matter Degeneration Preceding the Onset of Dementia." Alzheimer's \& Dementia 11 (5) (May): 485-493.e2. doi:10.1016/j.jalz.2014.04.518.

\section{Published Version}

doi:10.1016/j.jalz.2014.04.518

\section{Permanent link}

http://nrs.harvard.edu/urn-3:HUL.InstRepos:28538487

\section{Terms of Use}

This article was downloaded from Harvard University's DASH repository, and is made available under the terms and conditions applicable to Open Access Policy Articles, as set forth at http:// nrs.harvard.edu/urn-3:HUL.InstRepos:dash.current.terms-of-use\#OAP

\section{Share Your Story}

The Harvard community has made this article openly available.

Please share how this access benefits you. Submit a story.

\section{Accessibility}


Published in final edited form as:

Alzheimers Dement. 2015 May ; 11(5): 485-493.e2. doi:10.1016/j.jalz.2014.04.518.

\section{Widespread white matter degeneration preceding the onset of dementia}

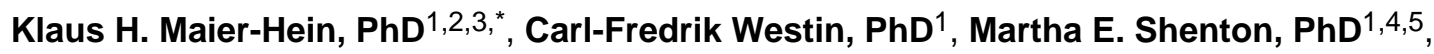
Michael W. Weiner, MD ${ }^{6}$, Ashish Raj, PhD ${ }^{7}$, Philipp Thomann, MD ${ }^{8}$, Ron Kikinis, MD ${ }^{1}$, Bram Stieltjes, $\mathrm{MD}, \mathrm{PhD}^{3}$, and Ofer Pasternak, $\mathrm{PhD}^{1,4}$

${ }^{1}$ Department of Radiology, Brigham and Women's Hospital, Harvard Medical School, Boston, MA, USA

${ }^{2}$ Division of Medical and Biological Informatics, German Cancer Research Center, Heidelberg, Germany

${ }^{3}$ Quantitative imaging-based disease characterization, German Cancer Research Center, Heidelberg, Germany

${ }^{4}$ Department of Psychiatry, Brigham and Women's Hospital, Harvard Medical School, Boston, MA, USA

${ }^{5}$ Health Scientist, Veterans Affairs Boston Healthcare System, Brockton Division, Brockton, MA, USA

${ }^{6}$ Center for Imaging of Neurodegenerative Disease, San Francisco VA Medical Center, University of California, San Francisco, USA

${ }^{7}$ Image Data Evaluation and Analytics Lab (IDEAL), Weill Cornell Medical College, New York, NY, USA

${ }^{8}$ Department of General Psychiatry, Section of Geriatric Psychiatry, Ruprecht-Karls-University, Heidelberg, Germany

\section{Abstract}

Background-Brain atrophy in subjects with mild cognitive impairment (MCI) introduces partial volume effects, limiting the sensitivity of diffusion tensor imaging to white matter microstructural degeneration. Appropriate correction isolates microstructural effects in MCI that might be precursors of Alzheimer's disease (AD).

Methods-Forty-eight participants (18 MCI, $15 \mathrm{AD}$ and 15 healthy controls) had MRI scans and clinical evaluations at baseline and follow-up after 36 month. $10 \mathrm{MCI}$ subjects were diagnosed

(C) 2014 Elsevier Inc. All rights reserved.

*Corresponding author: Dr. rer. nat. Klaus H. Maier-Hein (né Fritzsche), Computational Disease Analysis Group, Div. Medical and Biological Informatics (E130), German Cancer Research Center (DKFZ), Im Neuenheimer Feld 280, 69120 Heidelberg, Germany, k.maier-hein@dkfz.de, Phone: (+49) 6221/42-3545, Fax: (+49) 6221/42-2345.

Publisher's Disclaimer: This is a PDF file of an unedited manuscript that has been accepted for publication. As a service to our customers we are providing this early version of the manuscript. The manuscript will undergo copyediting, typesetting, and review of the resulting proof before it is published in its final citable form. Please note that during the production process errors may be discovered which could affect the content, and all legal disclaimers that apply to the journal pertain. 
with $\mathrm{AD}$ at follow-up and 8 remained MCI. Free-water corrected measures on the white matter skeleton were compared between groups.

Results-Free-water-corrected radial diffusivity, but not un-corrected radial diffusivity, was increased across the brain of the converted group compared to the non-converted group $(\mathrm{P}<0.05)$. The extent of increases was similar to that found comparing $\mathrm{AD}$ with controls.

Conclusion-Partial volume elimination reveals microstructural alterations preceding dementia. These alterations may prove to be an effective and feasible early biomarker of AD.

\section{Keywords}

Alzheimer's Disease; Mild Cognitive Impairment; Diffusion Tensor Imaging; Partial Volume Elimination; Free-water Imaging

\section{Background}

Magnetic resonance imaging (MRI) can identify pathological brain alterations associated with Alzheimer's disease (AD) (1). These include macrostructural effects such as gray matter (GM) and white matter (WM) atrophy, as well as microstructural WM alterations, such as Wallerian degeneration and compromised myelin integrity. GM atrophy can be identified using volumetric measures based on anatomical MRI, whereas microstructural alterations can be identified using diffusion MRI (dMRI), a method that is sensitive to micron scale tissue architecture (2).

Mild cognitive impairment (MCI) is often a pre-stage of Alzheimer's disease (AD) with a tenfold higher risk of developing dementia compared with healthy subjects (3). Therefore, the detection of imaging abnormalities in MCI may increase our understanding of the pathogenesis of $\mathrm{AD}$, as it may lead to improved early detection of patients at risk, and may thus provide an objective means for the assessment of medication(s) in early stage clinical trials. MRI studies have not yet been able to provide a robust diagnostic measure for the early stages of the disease (4). Specifically, dMRI studies report inconsistent findings when comparing patients with MCI and controls, even when using similar analysis methods (e.g. see tract based spatial statistics (TBSS) studies in Table S1).

The inconsistent findings in MCI might stem from the fact that the cognitive deficits of some patients may stabilize, improve, or progress to other dementias (5). Patients with MCI are also likely to have different underlying pathology, as for example some subjects have $\mathrm{AD}$ pathology in the form of neurofibrillary tangles of hyperphosphorylated tau (p-tau) and beta amyloid (A $\beta$ ) neuritic plaques (6), which may be responsible for the $\mathrm{MCI}$, while others may not have AD pathology, and their MCI is due to other pathologies (5). Therefore, patients with MCI do not comprise one clinical entity, which limits the predictive validity of MCI imaging abnormalities as a pre-dementia syndrome and/or as biomarkers of AD pathology (7-10).

Another limiting factor in the study of patients with $\mathrm{MCI}$ and $\mathrm{AD}$ is the influence of brain atrophy on the sensitivity of dMRI to microstructural changes. Part of the atrophy is likely age associated, since atrophy also appears in normal aging (11). Moreover, many structural 
MRI studies have demonstrated increased GM atrophy (e.g., hippocampi, entorhinal cortex, amygdala) and WM atrophy (e.g., Corpus callosum) in patients with AD or MCI in comparison with age-matched controls $(12,13)$. Atrophy in subjects with MCI might be an early manifestation of AD pathology (14). Nevertheless, atrophy has limited specificity as a pre-curser of $\mathrm{AD}$, as it may appear both in subjects with $\mathrm{MCI}$ who will develop $\mathrm{AD}$ and also appear in those who will not, as well as in patients with other brain disorders. Atrophy introduces macroscopic morphological changes in WM, such as loss of volume, reduced cellular density and increased extracellular space (15), which due to the limited resolution of dMRI, increases the influence of partial volume between different tissues (16). Unless these macroscopic morphological changes are accounted for, they decrease dMRI specificity and lead to difficulties in the interpretation of derived measurements. Thus, the partial volume effects associated with variable amounts of atrophy may have affected the interpretation of previous data (16). This, in conjunction with the group heterogeneity, might explain the diversity of previous findings regarding dMRI alterations in MCI.

Our aim is to decouple macroscopic and microscopic effects and obtain dMRI derived microstructural precursors of dementia by studying a sample of subjects diagnosed with MCI who were followed for 3 years after an initial MRI scan. Within this period, some subjects developed dementia and were diagnosed with $\mathrm{AD}$, while others did not. By comparing these two subsets of MCI subjects, we were able to identify abnormalities that may constitute an early precursor of $\mathrm{AD}$. These abnormalities were revealed only when controlling for partial-volume effects by applying the free-water imaging method (17), which corrects each image voxel for contamination from freely diffusing extracellular water molecules. The method separately models the contribution of such molecules from water molecules that are in the vicinity of cellular membranes such as axonal membrane and myelin sheath (18). By eliminating the extracellular contribution, the method accounts for partial-volume that could be introduced by atrophy, and makes it possible to identify whether or not microstructural changes, such as WM degeneration occur (16).

\section{Methods}

\subsection{Study populations}

This study comprised 48 subjects including 18 patients diagnosed with MCI, 15 patients diagnosed with $\mathrm{AD}$, and 15 age-matched healthy control subjects (HC). A demographic and clinical characterization of the groups can be found in Table 1. Group effects were evaluated using analysis of deviance for the generalized linear model implemented in MathWorks Matlab (R2012a, 7.14.0.739). Global cognitive deficits were assessed using the mini-mental state examination (MMSE). Cognitive performance was investigated with a standardized extensive neuropsychological test battery (CERAD). Subjects were classified as healthy if they scored within the age- and education-adjusted norm with respect to each of the CERAD subtests. The MCI diagnosis was defined by Levy's criteria of aging-associated cognitive decline (19) (see supplement S1). Mild to moderate AD was defined by the NINCDSADRDA-criteria. The clinical evaluation of all subjects included ascertainment of personal and family history, as well as physical, neurological, and neuropsychological examinations. Those with a history of ischemic heart disease, cancer, extensive macro- and 
microangiopathic changes on MRI, and severe cerebrovascular risk factors (e.g. instable hypertension or poorly controlled diabetes) were excluded. An MRI scan and a clinical evaluation took place at the time of recruitment. Clinical re-evaluation of the patients with MCI was performed three years after inclusion in the study (range 35-37 months). MRI was not acquired during re-evaluation. During these 3 years, 10 patients with MCI diagnostically converted to $\mathrm{AD}$ (MCI-c), while the remaining 8 patients with MCI maintained stable (MCI$\mathrm{nc}$ ). The local institutional review board approved all study protocols, written informed consent was obtained from all patients or their legal guardians.

\subsection{Image acquisition and preprocessing}

Imaging (MPRAGE and dMRI) was performed on a 1.5T clinical scanner. The acquisition and preprocessing protocol is described in the supplements (S2).

\subsection{Diffusion MRI processing}

The diffusion tensor imaging (DTI) model was fitted using the tensor toolkit (https:// gforge.inria.fr/projects/ttk) and used to quantify fractional anisotropy (FA), radial diffusivity (RD), and axial diffusivity (AXD). The AXD and RD measures, in particular, have been shown to be associated with microstructural changes that are related to WM degeneration (20).

In addition to the DTI model, we analyzed the data using the free-water imaging model (17), which assumes a separate free-water compartment with a fixed diffusivity of $3 \times 10^{-5}$ $\mathrm{mm}^{2} / \mathrm{sec}$ (the diffusion coefficient of free-water at body temperature). Mapping the fractional volume of the free-water compartments provides a free-water map (FW). A second compartment (the tissue compartment) fits the remaining signal, which originates from intra- and extracellular water molecules in the proximity of cellular membranes, i.e., from brain tissue (18). The tissue compartment is modeled by a diffusion tensor, and therefore yields the same type of maps as the DTI method, only now these maps are corrected for contamination by a freely diffusing extracellular compartment (FA-cor, RDcor, and AXD-cor), and are thus expected to be more specific to WM degeneration than the measures derived from DTI alone $(16,18)$. The free-water model was implemented in mitk (http://www.mitk.org). Recently, free-water corrected maps have shown increased specificity to microstructural changes with increased sensitivity to changes that occur in MCI $(21,22)$ and normal aging, compared with non-corrected measures.

In order to detect global patterns of WM degeneration, we aligned all subjects to a common space using a two-step registration approach directly on the tensor images using DTITK (http://www.nitrc.org/projects/dtitk). First, a template was bootstrapped (23), using the IXI aging template (24). Then, a population-specific template space was created by means of affine and diffeomorphic registration (23). We used TBSS (25) to build a common WM skeleton from FA images derived from the coregistered tensor images. We then mapped all dMRI measures (both with and without free-water elimination) onto the skeleton. 


\subsection{Volumetric measures}

Cortical reconstruction and volumetric segmentation was performed using Freesurfer (26).

The total GM (both cortical and sub-cortical) and WM volumes, normalized by the intracranial volume, were computed and the quality of the segmentation was visually evaluated for gross registration mistakes, resulting with the exclusion of one AD subject from the volumetric analysis.

\subsection{Statistical analysis}

Clinical scores were analyzed by means of t-tests and analysis of variance (ANOVA) using Stata 9 (StataCorp LP).

TBSS group comparisons between the $\mathrm{AD}$ and $\mathrm{HC}$ groups and the $\mathrm{MCI}-\mathrm{c}$ and $\mathrm{MCI}-\mathrm{nc}$ groups, with age as a covariate, were performed using threshold-free cluster enhancement (27) via a non-parametric test with 5000 permutations (Randomize, FSL), and a significance threshold of $\mathrm{p}=0.05$ corrected for multiple comparisons. The group comparisons were done separately for each diffusivity measure (either free-water corrected or not). We tested whether the GM or WM volume is correlated with FW using a Pearson Correlation test between the respective volumes and the mean FW over the entire WM skeleton of each subject. We tested for group effects in the GM and WM volumes using an analysis of deviance for the generalized linear model as implemented in MathWorks Matlab (R2012a, 7.14.0.739), with the GM and WM volumes being modeled as dependent and the group labels and age as independent variables.

\section{Results}

\subsection{Clinical scores}

The MMSE scores and statistical analysis of the different groups at recruitment and followup are presented in the supplements (S3). At time of recruitment, there was no significant mean MMSE score difference between the HC group and the MCI-c as well as the MCI-nc groups.

\subsection{Free-water (FW) maps}

Many of the WM skeleton voxels in the AD group were found to have higher values of FW when compared to the HC group (Fig. 1a). Significant $(\mathrm{p}<0.05)$ differences were identified in areas including the frontal, temporal, and parietal lobes, bilaterally, in an equal measure. Comparing the MCI-c and MCI-nc groups showed no significant differences in FW.

\subsection{Association between FW values and total GM volume}

Comparing the average FW estimates on the WM skeleton with the total GM and WM volumes tested the association between FW content in the WM and atrophic brain processes. While WM volume did not exhibit a significant group effect $(\mathrm{F}(41)=1.0721 ; \mathrm{p}=0.38269)$, significant group effects were found for the GM volume $(F(41)=6.5542 ; \mathrm{p}<10-3)$ and $F W$ $(F(41)=8.628 ; \mathrm{p}<10-4)$. Further analysis revealed that the AD group had significantly increased mean FW comparing with the HC group ( $\mathrm{t}(26)=-3.03 ; \mathrm{p}<10^{-2}$; Fig. 1b). Similarly, total GM volume was significantly decreased between the AD group and the HC 
group $\left(\mathrm{t}(26)=3.42 ; \mathrm{p}<10^{-2} ;\right.$ Fig. 1c). No significant differences were identified when comparing the mean FW $(\mathrm{t}(14)=-0.88 ; \mathrm{p}=0.393 ;$ Fig. $1 \mathrm{~b})$ or total $\mathrm{GM}$ volume $(\mathrm{t}(14)=0.74$; $\mathrm{p}=0.471 ;$ Fig. 1c) between the MCI-c and MCI-nc groups.

Correlating the average FW estimates on the WM skeleton for all subjects (all groups combined $\mathrm{n}=46$ ) with their respective total GM volumes, we found a highly significant negative correlation (Fig. 1d; Pearson's R $=-0.6693 ; \mathrm{p}<10^{-6}$ ), i.e., the more GM atrophy present, the higher were the FW values. WM volume did not correlate significantly with FW (Pearson's $\mathrm{R}=-0.2195 ; \mathrm{p}=0.1428$ ), nor did it correlate with GM volume (Pearson's $\mathrm{R}=$ $0.0516 ; \mathrm{p}=0.7332$ ).

\subsection{Diffusion MRI analysis}

Having established that the free-water measure in the WM of this population is associated with GM atrophy, we next investigate how the free-water correction affected the identification of significant group differences of DTI measures that are indicative of microstructural WM degeneration.

Radial diffusivity (RD)—Comparison of the WM skeleton between AD and HC (Fig. 2) demonstrated that the AD group had a widespread significant increase of RD. Controlling for partial-volume by using free-water elimination had little or no impact on these results, as the significant group differences were apparent in both the non-corrected (RD, Fig. 2a) and the free-water corrected (RD-cor, Fig. $2 b)$ measures $(p<0.05)$. This was despite the fact that the AD group had significantly higher FW values than the HC group (see above, and Fig. 2). The two corrected and non-corrected RD measures overlapped, with a small fraction of voxels on the WM skeleton that was identified by only one of the measures. The areas where $\mathrm{RD}$ and RD-cor overlap included the frontal, temporal, and parietal lobes, bilaterally, in an equal measure, similar to the extent of increased FW reported above. These areas included the medial temporal, retrosplenial regions, anterior commissure, corona radiata, internal capsule, corticopontine tracts, cerebral peduncle, striatum, and precentral gyrus. In a recent study microstructural differences independent of volumetric changes were found in these areas in $\mathrm{AD}$ (28). The small areas that were identified using only RD or only RD-cor were scattered throughout the brain without a clear pattern.

Comparison of the WM skeleton between the MCI-c and MCI-nc groups (Fig. 3) showed that the MCI-c group had significantly increased non-corrected RD (Fig. 3a) only within a small subset of voxels, all within the Corpus callosum. On the other hand, correcting for free-water revealed widespread significantly increased RD-cor values $(\mathrm{p}<0.05)$ for the MCIc group (Fig. 3b). In other words, controlling for atrophy using the free-water elimination method drastically increased the number of areas where WM degeneration in the MCI-c group was identified. The areas identified with the RD-cor measure included the areas that were identified using the non-corrected RD values. The extent of increased RDcor identified for the MCI-c group was similar to the extent of the increased RD-cor identified for the $\mathrm{AD}$ group (compare Fig 2b with Fig. 3b), although the extent of findings in the MCI-c group was slightly reduced, and in particular the frontal lobe was less affected. Specifically, the orbitofrontal cortex was bilaterally affected in MCI-c, with a predominance of the left 
hemisphere. The cingulate gyrus was affected bilaterally in an equal measure. The rightsided dorsolateral prefrontal cortex was spared in MCI-c, whereas the left side was affected. In $\mathrm{AD}$, both sides were affected in an equal measure.

Other diffusivities-Significant differences ( $\mathrm{p}<0.05$ ) between the AD and $\mathrm{HC}$ groups in FA, FA-cor, AXD, and AXD-cor were less extensive than RD-cor (not shown). Comparing the MCI-c and MCI-nc groups showed significantly lower FA-cor ( $\mathrm{p}<0.05)$ only within the Corpus callosum, and no significant differences in FA, AXD, or AXD-cor.

\section{Discussion}

Our results indicate that widespread WM microstructural abnormalities, detected after correction for partial volume effects, are associated with the development of dementia due to AD. These abnormalities are likely indicative of WM degeneration and were found in patients with MCI, preceding the onset of dementia, as well as in subjects who are already diagnosed with $\mathrm{AD}$. These early signs of neurodegenerative processes were found across the brain.

The most important finding in our study is that there was a widespread and significant RDcor increase in the MCI-c group comparing with the MCI-nc group. This increase was similar in extent to that evinced when comparing the $\mathrm{AD}$ group with healthy controls, suggesting that neurodegenerative processes are present even before the onset of dementia in those subjects with $\mathrm{MCI}$ who will convert to $\mathrm{AD}$ in the future. DTI Findings in the early stages of AD and in MCI have been reported previously (29, 30). Of particular note are our findings in the frontal lobe. It is generally accepted that the destructive process during the clinical course of $\mathrm{AD}$ follows a characteristic pattern, in which the frontal lobe is involved in the isocortical stage, with frontal lobe-related neurocognitive deterioration appearing only in the late stages of $\mathrm{AD}$ and absent in the MCI subjects (6). Accordingly, we find reduced frontal lobe involvement in MCI-c comparing with AD. Nevertheless, the frontal lobe areas that show increased RD-cor in the MCI-c group strongly suggest that this pathology precedes the manifestation of frontal lobe-related symptoms (e.g. inhibition) that are absent in MCI. DTI Findings in the early stages of AD and in MCI have been reported previously $(29,30)$. However, studies that did not separate MCI-c from MCI-nc are limited in their reproducibility (see Table S1), and studies that performed this separation were either limited to the analysis of regions of interest $(9,31,32)$ or used supervised learning for classification $(33,34)$. Thus, none of these studies has thus far been able to demonstrate the extent of microstructural alterations in subjects with MCI that will develop dementia. One previous study used TBSS for the examination of group differences between MCI-c and MCI-nc, but that study did not find a significant group difference and did not correct for partial volume (35). Here, by associating the FW measure with the GM volume we are able to demonstrate that atrophy related processes are likely affecting the ability to identify microstructural DTI differences in the WM of subjects with MCI and that following free-water elimination, such WM alterations are much more robustly identified. We did not find an association between GM and WM volumes, suggesting that at least in its early stages, atrophy mostly affects the extracellular volume and the density of white matter, which are not apparent in volumetric MRI measures of WM (15), but are apparent in the free-water measure. This is important 
since it will potentially improve current imaging-based $\mathrm{AD}$ prediction methods, which directly benefit from imaging markers that are more sensitive and more specific to $A D$ pathology.

Atrophy is known to be prominent in AD-type dementia (1), and in agreement with this observation, we found highly elevated GM atrophy for $\mathrm{AD}$ patients compared with the other groups. A previous study in an aging population demonstrated that dMRI quantities such as $\mathrm{RD}$ and $\mathrm{AXD}$ are affected by partial-volume and atrophy, with diffusivity measures increasing as atrophy is more severe (16). In that study, using free-water elimination to control for partial-volume effects eliminated group differences that were likely attributed to increased atrophy with aging, and did not reflect microstructural changes. In the current study, we similarly find that the AD subjects evince significantly increased FW comparing with age-matched controls, correlating with increased atrophy. However, unlike the aging study, in the current study there was a significant group difference even after the free-water correction (increased RD-cor). This suggests that in AD there are additional dMRI alterations that occur on a microstructural level. These additional findings are likely related to WM degeneration, which might appear in normal aging as well, but is known to be much more pronounced and wide-spread in subjects with $\mathrm{AD}(6)$.

In the comparison of the MCI-c and MCI-nc groups the RD-cor differences were revealed only following the free-water correction. This is opposite to previous free-water elimination studies, where the elimination of partial-volume effects served mostly to reduce group difference in the DTI corrected measures $(16,18)$. This difference can be explained by noting that we found a similar degree of GM atrophy between the MCI-c and MCI-nc groups, which means that variable amounts of partial-volume effects are expected in these datasets. The variable partial-volume effects dominate the signal, introducing a bias that prevents the identification of the much more subtle microstructural group differences. The free-water elimination effectively reduces this bias, making possible the identification of whether or not there are microstructural differences between the groups. As a result, the free-water eliminated DTI measures are more specific to microstructural processes that occur in brain tissue rather than in the extracellular space (18). In the data presented, we find that there are such microstructural changes between the MCI-c and MCI-nc groups (as well as between the $\mathrm{AD}$ and $\mathrm{HC}$ groups), whereas in previous studies on other type of populations, such microstructural changes were not found, or had a limited extent. Taken together, our results demonstrate that the free-water corrected RD (RD-cor), which decouples dMRI's microstructural effects from its macrostructural effects, provides better separability than the total volume of GM between the MCI-c and MCI-nc groups. While it is likely that regional GM volumetric measures might have increased prediction power, previous studies have demonstrated that combining conventional DTI measures with GM volumetric measures increases classification accuracy of MCI from healthy controls (36). We therefore suggest that the free-water corrected measures could be an important addition to GM atrophy measures in the prediction of the development of dementia caused by AD. This is also supported by recent studies where free-water corrected measures in preselected WM connections were associated with changes in behavioral measures related to $\mathrm{AD}$ (21, 22). 
We have demonstrated the importance of having a sample of subjects with dMRI acquisitions at baseline and a known future conversion to AD. Future longitudinal studies on larger numbers of patients with MCI, e.g., the diffusion MRI subset of the ADNI-2 dataset on which the follow-up information will eventually become available, will allow us to substantiate our findings. Another potential extension of our work would be the incorporation of the corrected diffusion measure into network analytics applied to similar patient groups. This could potentially be translated to the clinical domain by creating a pool of normative controls and detecting and comparing degeneration patterns on a single-patient basis (37). This information may prove useful in the individual management of patients with MCI, and possibly in the development of targeted drugs.

\section{Supplementary Material}

Refer to Web version on PubMed Central for supplementary material.

\section{Acknowledgements}

Conflicts/Funding Sources

This work was supported in part by National Institute of Health grants R01MH074794, P41RR013218 and P41EB015902; by the German Research Foundation (DFG), grant\# ME 833/15-1 (to K.H.MH and B.S.); and by a NARSAD Young Investigator Grant from the Brain and Behavior Research Foundation (to O.P.).

\section{Abbreviations}

$\begin{array}{ll}\text { AXD } & \text { Axial diffusivity } \\ \text { AXD-cor } & \text { Free-water corrected axial diffusivity } \\ \text { dMRI } & \text { Diffusion MRI } \\ \text { DTI } & \text { Diffusion tensor imaging } \\ \text { FA } & \text { Fractional anisotropy } \\ \text { FA-cor } & \text { Free-water corrected fractional anisotropy } \\ \text { FW } & \text { Free-water } \\ \text { GM } & \text { Gray matter } \\ \text { HC } & \text { Healthy control subjects } \\ \text { MCI } & \text { Mild cognitive impairment } \\ \text { MCI-c } & \text { Mild cognitive impairment subjects with future conversion to Alzheimer's } \\ & \text { disease } \\ \text { MCI-nc } & \text { Mild cognitive impairment subjects with no future conversion to Alzheimer's } \\ \text { RD } & \text { disease } \\ \text { RD-cor } & \text { Radial diffusivity } \\ \text { FBSS } & \text { Tree-water corrected radial diffusivity } \\ & \end{array}$




\section{References}

1. Dubois B, Feldman HH, Jacova C, Dekosky ST, Barberger-Gateau P, Cummings J, et al. Research criteria for the diagnosis of Alzheimer's disease: revising the NINCDS-ADRDA criteria. Lancet neurology. 2007 Aug; 6(8):734-746.

2. Assaf Y, Pasternak O. Diffusion tensor imaging (DTI)-based white matter mapping in brain research: a review. Journal of molecular neuroscience : MN. 2008; 34(1):51-61. [PubMed: 18157658]

3. Mitchell AJ, Shiri-Feshki M. Rate of progression of mild cognitive impairment to dementia--metaanalysis of 41 robust inception cohort studies. Acta psychiatrica Scandinavica. 2009 Apr; 119(4): 252-265. [PubMed: 19236314]

4. Albert MS, DeKosky ST, Dickson D, Dubois B, Feldman HH, Fox NC, et al. The diagnosis of mild cognitive impairment due to Alzheimer's disease: recommendations from the National Institute on Aging-Alzheimer's Association workgroups on diagnostic guidelines for Alzheimer's disease. Alzheimer's \& dementia : the journal of the Alzheimer's Association. 2011 May; 7(3):270-279.

5. Petersen RC. Mild cognitive impairment as a diagnostic entity. Journal of internal medicine. 2004 Sep; 256(3):183-194. [PubMed: 15324362]

6. Braak H, Braak E. Neuropathological stageing of Alzheimer-related changes. Acta neuropathologica. 1991; 82(4):239-259. [PubMed: 1759558]

7. van Bruggen T, Stieltjes B, Thomann PA, Parzer P, Meinzer HP, Fritzsche KH. Do Alzheimerspecific microstructural changes in mild cognitive impairment predict conversion? Psychiatry research. 2012 Aug-Sep;203(2-3):184-193. [PubMed: 22947309]

8. Killiany RJ, Gomez-Isla T, Moss M, Kikinis R, Sandor T, Jolesz F, et al. Use of structural magnetic resonance imaging to predict who will get Alzheimer's disease. Annals of neurology. 2000 Apr; 47(4):430-439. [PubMed: 10762153]

9. Mielke MM, Okonkwo OC, Oishi K, Mori S, Tighe S, Miller MI, et al. Fornix integrity and hippocampal volume predict memory decline and progression to Alzheimer's disease. Alzheimer's \& dementia : the journal of the Alzheimer's Association. 2012; 8(2):105-113.

10. Rosenberg PB, Hillis AE. Biomarkers for Alzheimer's disease: ready for the next step. Brain : a journal of neurology. 2009 Aug; 132(Pt 8):2002-2004. [PubMed: 19617196]

11. Scahill RI, Frost C, Jenkins R, Whitwell JL, Rossor MN, Fox NC. A longitudinal study of brain volume changes in normal aging using serial registered magnetic resonance imaging. Archives of neurology. 2003 Jul; 60(7):989-994. [PubMed: 12873856]

12. Du AT, Schuff N, Amend D, Laakso MP, Hsu YY, Jagust WJ, et al. Magnetic resonance imaging of the entorhinal cortex and hippocampus in mild cognitive impairment and Alzheimer's disease. Journal of neurology, neurosurgery, and psychiatry. 2001 Oct; 71(4):441-447.

13. Hampel H, Teipel SJ, Alexander GE, Horwitz B, Teichberg D, Schapiro MB, et al. Corpus callosum atrophy is a possible indicator of region- and cell type-specific neuronal degeneration in Alzheimer disease: a magnetic resonance imaging analysis. Archives of neurology. 1998 Feb; 55(2):193-198. [PubMed: 9482361]

14. Jack CR Jr, Petersen RC, Xu YC, O'Brien PC, Smith GE, Ivnik RJ, et al. Prediction of AD with MRI-based hippocampal volume in mild cognitive impairment. Neurology. 1999 Apr 22; 52(7): 1397-1403. [PubMed: 10227624]

15. Fjell AM, Westlye LT, Greve DN, Fischl B, Benner T, van der Kouwe AJ, et al. The relationship between diffusion tensor imaging and volumetry as measures of white matter properties. NeuroImage. 2008 Oct 1; 42(4):1654-1668. [PubMed: 18620064]

16. Metzler-Baddeley C, O'Sullivan MJ, Bells S, Pasternak O, Jones DK. How and how not to correct for CSF-contamination in diffusion MRI. NeuroImage. 2012 Jan 16; 59(2):1394-1403. [PubMed: 21924365] 
17. Pasternak O, Sochen N, Gur Y, Intrator N, Assaf Y. Free water elimination and mapping from diffusion MRI. Magnetic resonance in medicine : official journal of the Society of Magnetic Resonance in Medicine / Society of Magnetic Resonance in Medicine. 2009 Sep; 62(3):717-730.

18. Pasternak O, Westin CF, Bouix S, Seidman LJ, Goldstein JM, Woo TU, et al. Excessive extracellular volume reveals a neurodegenerative pattern in schizophrenia onset. The Journal of neuroscience : the official journal of the Society for Neuroscience. 2012 Nov 28; 32(48):1736517372. [PubMed: 23197727]

19. Levy R. Aging-associated cognitive decline. Working Party of the International Psychogeriatric Association in collaboration with the World Health Organization. International psychogeriatrics / IPA. 1994 Spring;6(1):63-68. [PubMed: 8054494]

20. Song SK, Sun SW, Ju WK, Lin SJ, Cross AH, Neufeld AH. Diffusion tensor imaging detects and differentiates axon and myelin degeneration in mouse optic nerve after retinal ischemia. NeuroImage. 2003 Nov; 20(3):1714-1722. [PubMed: 14642481]

21. Metzler-Baddeley C, Hunt S, Jones DK, Leemans A, Aggleton JP, O'Sullivan MJ. Temporal association tracts and the breakdown of episodic memory in mild cognitive impairment. Neurology. 2012 Dec 4; 79(23):2233-2240. [PubMed: 23175726]

22. Metzler-Baddeley C, Jones DK, Steventon J, Westacott L, Aggleton JP, O'Sullivan MJ. Cingulum microstructure predicts cognitive control in older age and mild cognitive impairment. The Journal of neuroscience : the official journal of the Society for Neuroscience. 2012 Dec 5; 32(49):1761217619. [PubMed: 23223284]

23. Zhang H, Yushkevich PA, Rueckert D, Gee JC. Unbiased white matter atlas construction using diffusion tensor images. Medical image computing and computer-assisted intervention : MICCAI International Conference on Medical Image Computing and Computer-Assisted Intervention. 2007; 10(Pt 2):211-218.

24. Zhang, H.; Yushkevich, P.; Rueckert, D.; Gee, J. A Computational White Matter Atlas for Aging with Surface-Based Representation of Fasciculi. In: Fischer, B.; Dawant, B.; Lorenz, C., editors. Biomedical Image Registration: Springer Berlin Heidelberg. 2010. p. 83-90.

25. Smith SM, Jenkinson M, Johansen-Berg H, Rueckert D, Nichols TE, Mackay CE, et al. Tractbased spatial statistics: voxelwise analysis of multi-subject diffusion data. NeuroImage. $2006 \mathrm{Jul}$ 15; 31(4):1487-1505. [PubMed: 16624579]

26. Fischl B, Salat DH, Busa E, Albert M, Dieterich M, Haselgrove C, et al. Whole brain segmentation: automated labeling of neuroanatomical structures in the human brain. Neuron. 2002 Jan 31; 33(3):341-355. [PubMed: 11832223]

27. Smith SM, Nichols TE. Threshold-free cluster enhancement: addressing problems of smoothing, threshold dependence and localisation in cluster inference. NeuroImage. 2009 Jan 1; 44(1):83-98. [PubMed: 18501637]

28. Canu E, McLaren DG, Fitzgerald ME, Bendlin BB, Zoccatelli G, Alessandrini F, et al. Microstructural diffusion changes are independent of macrostructural volume loss in moderate to severe Alzheimer's disease. Journal of Alzheimer's disease : JAD. 2010; 19(3):963-976.

29. Zhang Y, Schuff N, Jahng GH, Bayne W, Mori S, Schad L, et al. Diffusion tensor imaging of cingulum fibers in mild cognitive impairment and Alzheimer disease. Neurology. 2007 Jan 2; 68(1):13-19. [PubMed: 17200485]

30. Fellgiebel A, Wille P, Muller MJ, Winterer G, Scheurich A, Vucurevic G, et al. Ultrastructural hippocampal and white matter alterations in mild cognitive impairment: a diffusion tensor imaging study. Dementia and geriatric cognitive disorders. 2004; 18(1):101-108. [PubMed: 15087585]

31. Fellgiebel A, Dellani PR, Greverus D, Scheurich A, Stoeter P, Muller MJ. Predicting conversion to dementia in mild cognitive impairment by volumetric and diffusivity measurements of the hippocampus. Psychiatry research. 2006 Apr 30; 146(3):283-287. [PubMed: 16530394]

32. Douaud G, Menke RA, Gass A, Monsch AU, Rao A, Whitcher B, et al. Brain microstructure reveals early abnormalities more than two years prior to clinical progression from mild cognitive impairment to Alzheimer's disease. The Journal of neuroscience : the official journal of the Society for Neuroscience. 2013 Jan 30; 33(5):2147-2155. [PubMed: 23365250] 
33. Oishi K, Akhter K, Mielke M, Ceritoglu C, Zhang J, Jiang H, et al. Multi-modal MRI analysis with disease-specific spatial filtering: initial testing to predict mild cognitive impairment patients who convert to Alzheimer's disease. Frontiers in neurology. 2011; 2:54. [PubMed: 21904533]

34. Hahn K, Myers N, Prigarin S, Rodenacker K, Kurz A, Forstl H, et al. Selectively and progressively disrupted structural connectivity of functional brain networks in Alzheimer's disease - revealed by a novel framework to analyze edge distributions of networks detecting disruptions with strong statistical evidence. NeuroImage. 2013 Nov 1.81:96-109. [PubMed: 23668966]

35. Haller S, Nguyen D, Rodriguez C, Emch J, Gold G, Bartsch A, et al. Individual prediction of cognitive decline in mild cognitive impairment using support vector machine-based analysis of diffusion tensor imaging data. Journal of Alzheimer's disease : JAD. 2010; 22(1):315-327.

36. Zhang Y, Schuff N, Camacho M, Chao LL, Fletcher TP, Yaffe K, et al. MRI markers for mild cognitive impairment: comparisons between white matter integrity and gray matter volume measurements. PloS one. 2013; 8(6):e66367. [PubMed: 23762488]

37. Bouix S, Pasternak O, Rathi Y, Pelavin PE, Zafonte R, Shenton ME. Increased gray matter diffusion anisotropy in patients with persistent post-concussive symptoms following mild traumatic brain injury. PloS one. 2013; 8(6):e66205. [PubMed: 23776631] 


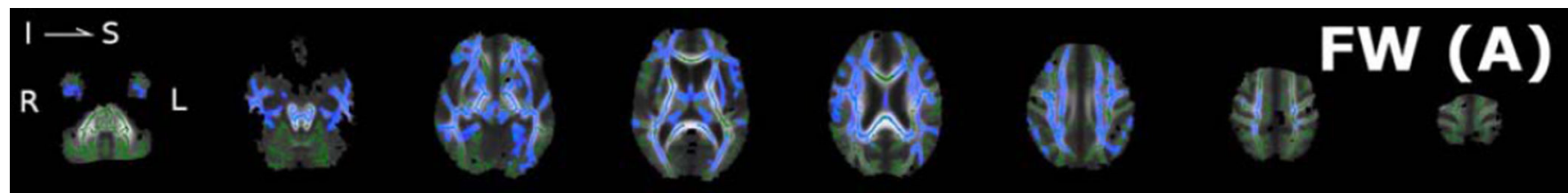

$\mathrm{R} \longrightarrow \mathrm{L}$

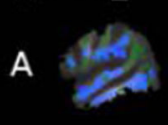

P
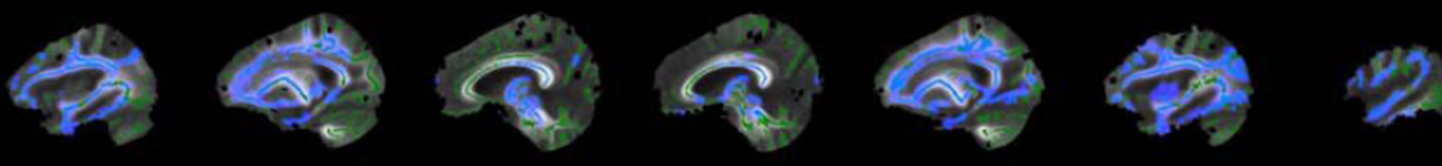

$A \rightarrow P$

$R$ Sers

L
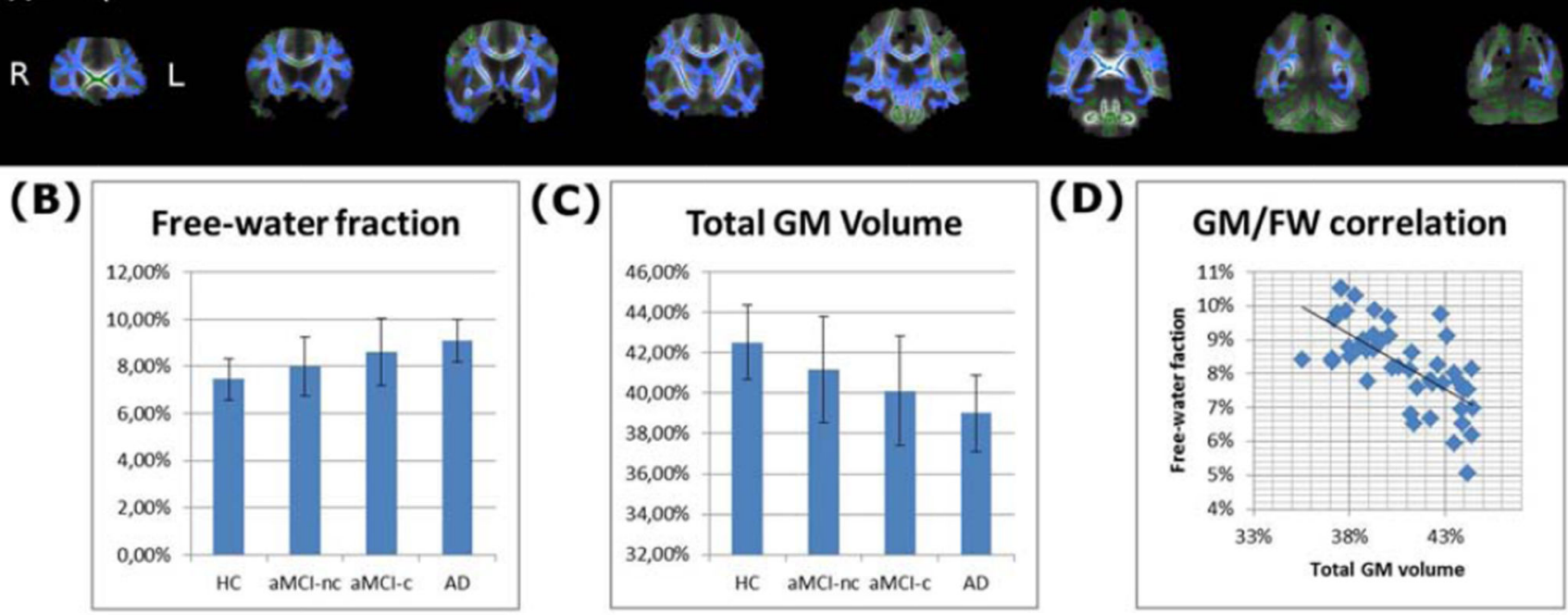

(D)

\section{GM/FW correlation}

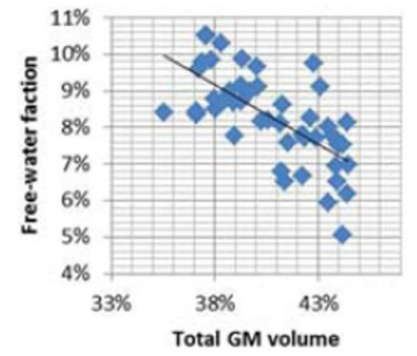

Figure 1. Association between free-water (FW) and atrophy in AD and MCI

(A) Widespread significantly increased FW is found between the AD group and the healthy control (HC) group ( $\mathrm{p}<0.05$ in blue, on top of the white matter skeleton in green). There were no significant differences between the MCI-nc and MCI-c groups (not shown). A gradual increase along the progression of $\mathrm{AD}$ in the mean $\mathrm{FW}$ over the entire skeleton was observed comparing the 4 groups (B). At the same time a gradual decrease in the GM volume was observed (C). (D) The FW values were highly negatively correlated with the GM volume (Pearson's $\mathrm{R}=-0.6693 ; \mathrm{p}<10^{-6}$ ), suggesting a widespread pattern of atrophyrelated changes. 


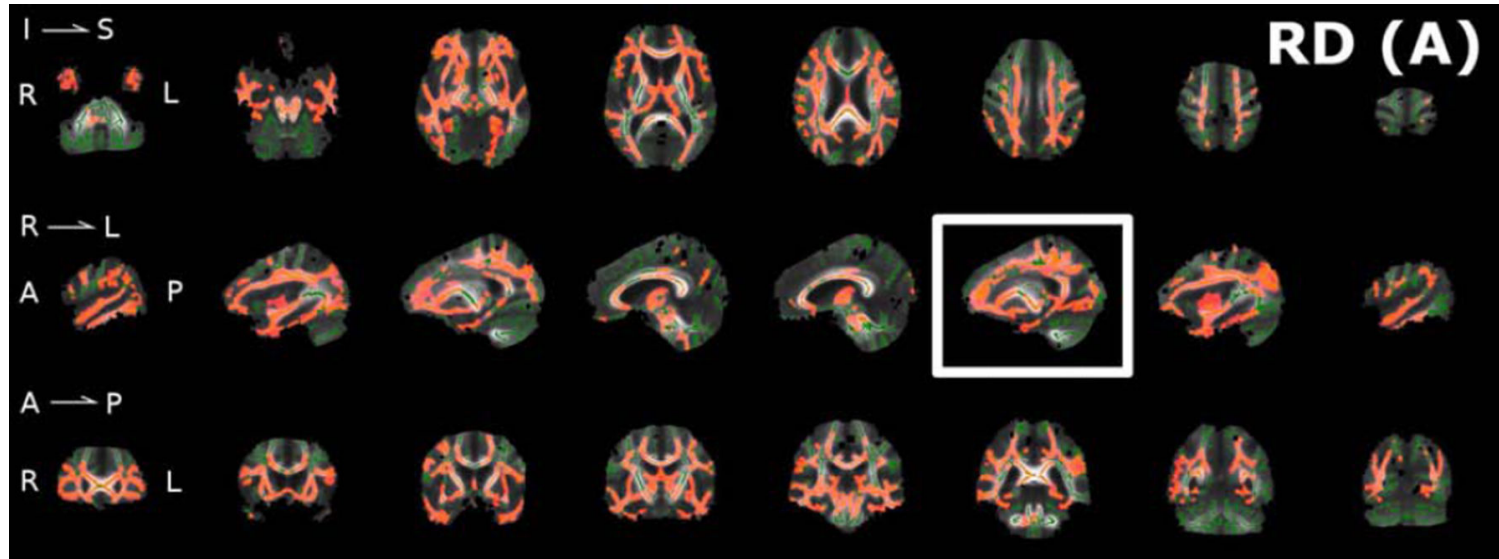

$1 \rightarrow S$
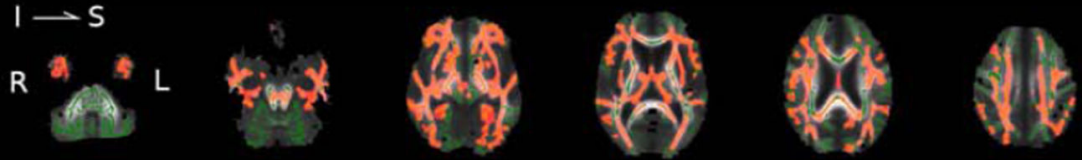

RD-cor (B)

$R \rightarrow L$
$A$

$P$
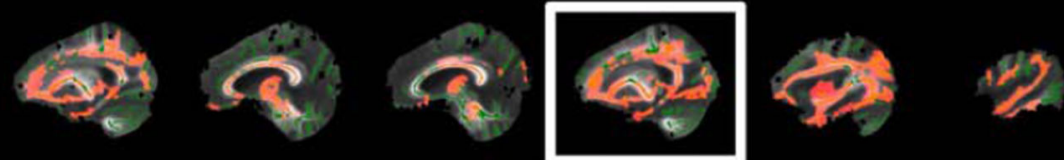

$A \rightarrow P$

$R$ ax $L$ (2)
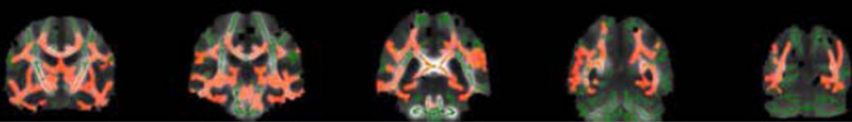

\section{RD (C)}

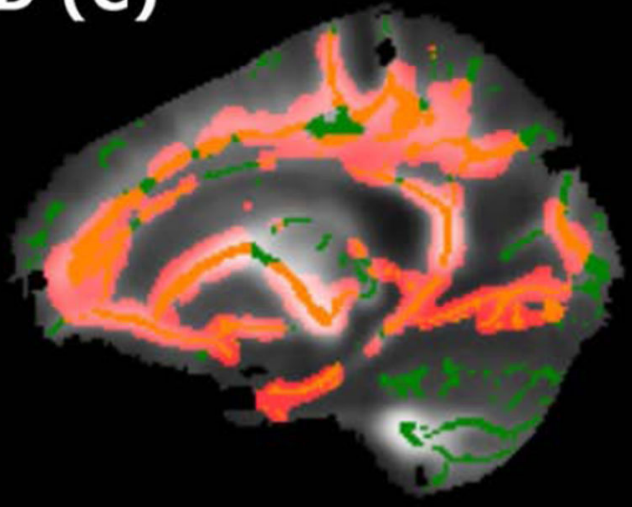

RD-cor (D)

Figure 2. White matter degeneration in Alzheimer's disease

Comparing patients diagnosed with $\mathrm{AD}$ and healthy controls reveals widespread significantly increased radial diffusivity (RD). Controlling for atrophy by using free-water elimination had little impact on the results as both conventional RD maps (A) and free-water corrected maps (RD-cor; B) identified overlapping brain areas, indicating extensive WM degeneration in the AD group. Significant results $(p<0.05)$ are colored red, on top of the white matter skeleton in green, with (C) and (D) showing enlarged versions of the sagittal slice marked by the white box. 

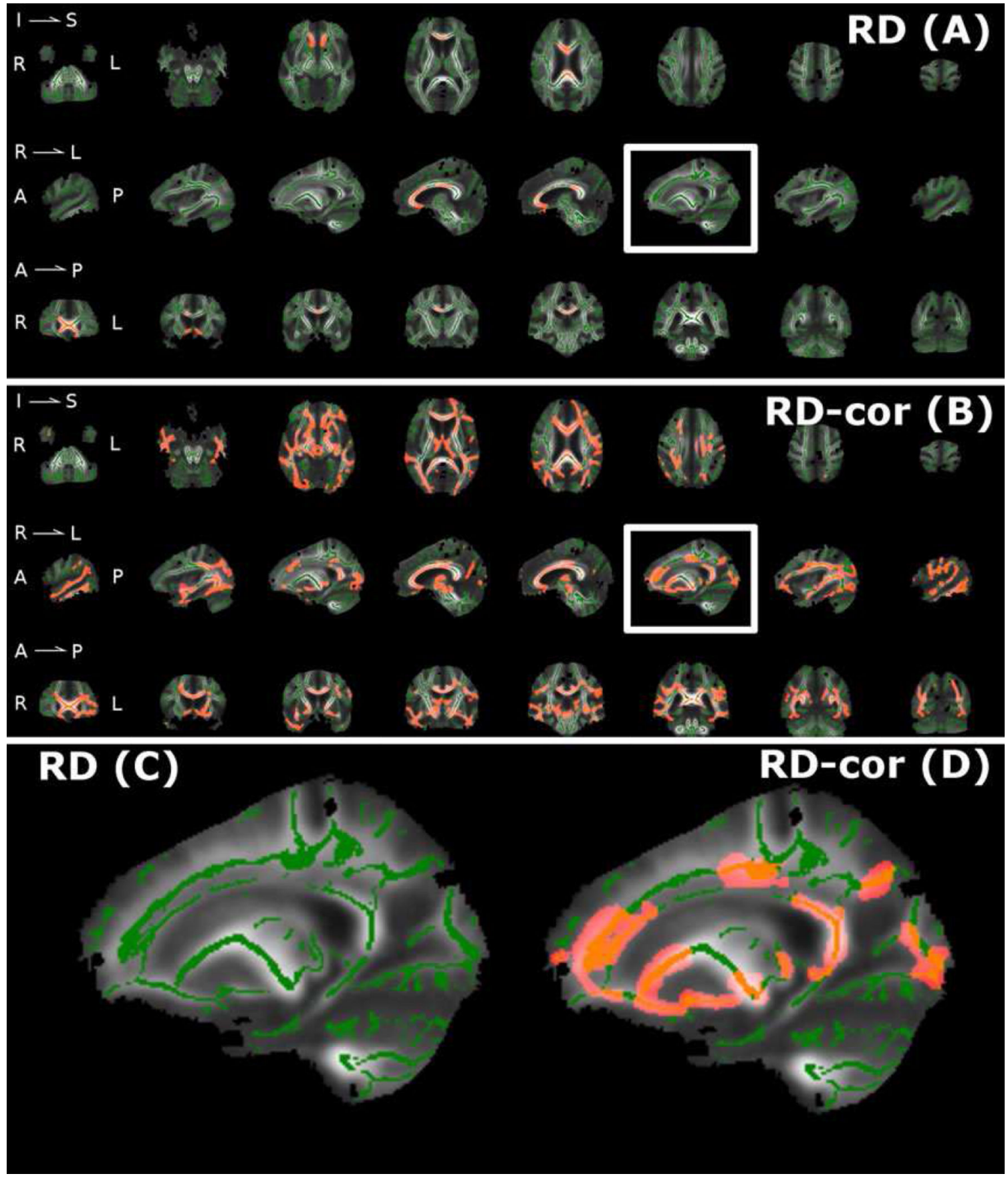

Figure 3. White matter degeneration in subjects with MCI that were later diagnosed with AD In a Comparison of subjects with MCI who were later diagnosed with AD (MCI-c) and subjects with MCI that did not develop dementia within a 3 year follow up (MCI-nc), the non-corrected RD measure (A) demonstrates significant increases only in a small subset of voxels in the Corpus callosum. Controlling for atrophy with the free-water corrected measure, RD-cor (B), reveals a widespread pattern of WM degeneration between the MCInc and MCI-c groups. Significant results $(\mathrm{p}<0.05)$ are colored red, on top of the white matter 
skeleton in green, with (C) and (D) showing enlarged versions of the sagittal slice marked by the white box. 


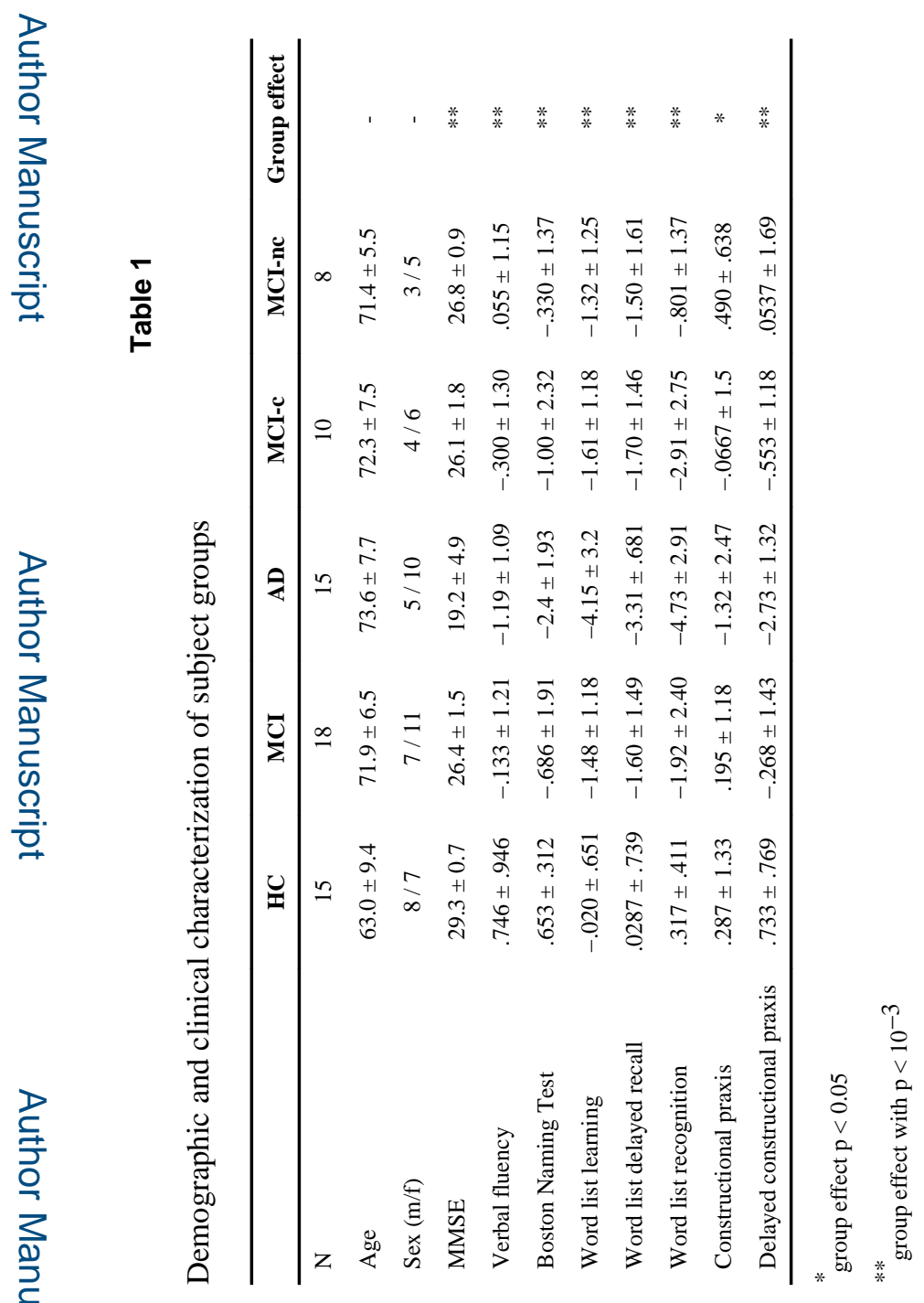

